\title{
Real Time Attendance Marking System
}

\author{
Riya John \\ Mar Baselios college of engineering \\ and technology Asst professor, \\ Dept of ECE \\ Trivandrum,India \\ Jeen Raju \\ Mar Baselios college of engineering and technology \\ Dept of ECE \\ Trivandrum,India
}

\author{
Akhilesh.s \\ Mar Baselios college of engineering \\ and technology Dept of ECE \\ Trivandrum,India
}

\author{
Gayathri Geetha Nair \\ Mar Baselios college of engineering \\ and technology Dept of ECE \\ Trivandrum,India
}

\author{
Krishnendhu.B \\ Mar Baselios college of engineering and technology \\ Dept of ECE \\ Trivandrum,India.
}

\begin{abstract}
Attendance management is an important procedure in an educational institution as well as in business organizations. Most of the available methods are time consuming and manipulative. The traditional method of attendance management is carried out in handwritten registers. Other than the manual method, there exist biometric methods like fingerprint and retinal scan, RFID tags, etc. All of these methods have disadvantages, therefore, in order to avoid these difficulties here, we introduce a new method for attendance management using deep learning technology. Using deep learning we can easily train a data-set. Real-time face algorithms are used and recognized faces of students in real-time while attending lectures. This system aims to be less time- consuming in comparison to the existing system of marking attendance.The program runs on anaconda flask server.Here real time image is captured using mobile phone camera. The faces on the image of the persons are then recognized and attendance is marked on an excel file.
\end{abstract}

Keywords:- Flask Server, Face Detection, Face Embedding, Face Recognition.

\section{INTRODUCTION}

Nowadays, technology has improved a lot but still, attendance management is a major issue. Attendance marking is necessary to evaluate the performance of the students and the employees. There exist many methods of attendance marking. The traditional method involves the signature on paper sheets or manual systems that require human sight. Many methods have been developed in order to improve the existing method. Considering the case of a class, taking manual attendance every day and every period is such a tedious, time-wasting process and requires a lot of human effort. Here the attendance will be carried out in the handwritten registers. It causes interruptions in the class, very error-prone and wastage of lot of resources. Attendance management is not only a problem within the classroom, but it also affect the business organization. Other than the manual method, there exist other methods that is biometric methods like fingerprint, retinal scan, and RFID tags and so on. While using RFID tag masquerading is easy for employees and in other method attendance of a group of people cannot be marked at the same time.

Attendance marking is effective only when it is realtime. The disadvantage of the previous methods can be trimmed down by employing facial recognition to mark the attendance. Face detection, which is a simple and easy task for humans is not so for computers. It has been regarded as the most complex and challenging problem in the field of computer vision due to large intra-class variations caused by the changes in facial appearance, lighting, and expression. Such variations result in the face distribution to be highly nonlinear and complex in any space that is linear to the original image space. Face detection is the process of identifying one or more human faces in images or videos. So we aim at making the attendance- taking process automatic and hands- free by employing facial recognition systems so it can automatically update digital attendance records in real- time. This will computerize the traditional method of attendance marking and thereby provides a better user interface. This can be achieved by using a technology called deep learning, which make use of neural networks to teach computer by observing data. The proposed system capture live images using mobile phone cameras and the face detection is done using a frontal face detector. The detected face are then converted to face embedding using facenet[6][9].A neural network is used for training process where 40 to 50 images per person are face embedded and trained. The detected face embedding are then predicted from the trained model for recognition process. This is then marked to an excel sheet as attendance.

\section{PREVIOUS WORKS}

One of the methods was to mark the attendance was by using a motion sensor and a camera which turns on when motion is detected by the sensor. Here the process is that when the sensor detects motion it automatically makes sure the camera is recording a video of the students and compares the input data with the data that is stored in the database. The defect of this system is that even the slightest movement will result in the camera to turn on[1]. The second method is based on deep learning face recognition 
where using Dlib which analysis the chin and nose of the person and compares it with the Rfid tag logged at that moment.This is a tedious task and takes a long time for processing[2]. The third method is the multi-face recognition where faces were detected for straight faces and faces at a distance of about $200 \mathrm{~cm}$. This process can be implemented to get the most faces at the point of time[3]. The fourth method is face recognition using raspberry pi where the face is recorded on the camera and compared with the stored data and the value preset and absent is marked according to the recognized face. This process can only recognize a few people at the time and due to lighting issues, it can't recognize a large number of people in the class[4].

\section{REAL TIME ATTENDANCE MARKING SYSTEM}

The proposed system workflow is as shown in Figure 1. In order to capture live image using mobile phone camera a web server is created. This image will be the input to the face recognition model. The server program which is combined with the face recognition model will be running in anaconda flask server.On browsing the server Internet Protocol (IP) address on mobile phone a window opens that enable the access to phone camera and capture the live image.This image is uploaded for further face recognition process, where faces are detected and their embeddings are created using facenet[8][9].The embedding values of the detected face are then predicted from the embedded values of trained data. After analyzing the data it marks the attendance with the respect to identifying faces in the images with values like a present and absent. This digital information is marked automatically to the name of the student without external help.

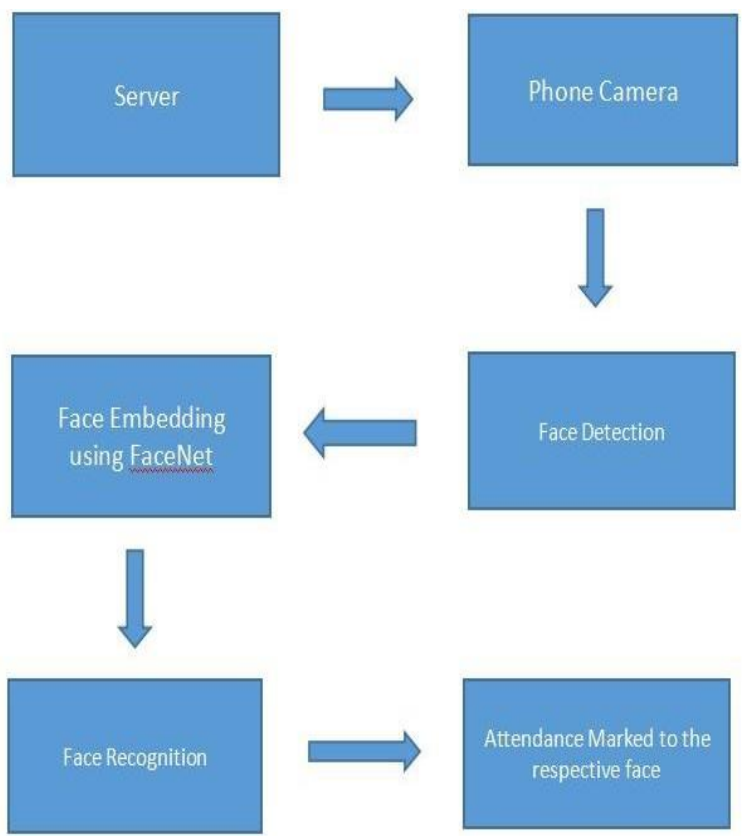

Fig 1:- Proposed Work-flow

\section{PROPOSED SYSTEM}

\section{Server}

To give the input from the phone camera a server is created in anaconda flask server. The server here is a computer program that provides functionality for other programs or devices clients(mobile phone).The server program is combined with a face recognition model .While the program is running on the system, by browsing the server IP address we can access the phone camera as shown in figure 2.The IP address identifies the location of a specific server that is connected to the internet. Here the mobile phone and the system running the program is connected to the same wifi.By choosing the camera option live image can be captured as shown in figure 3.This image is then uploaded as shown in figure 4 for further face detection and recognition

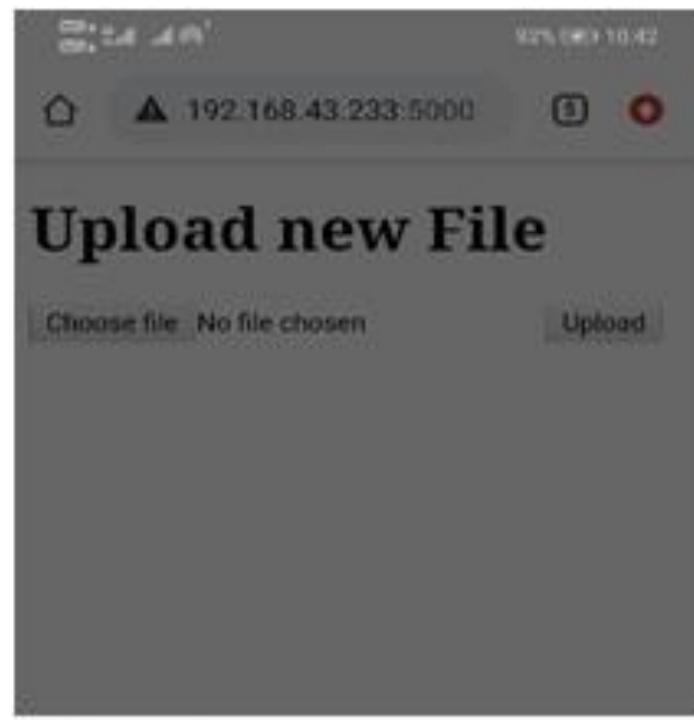

Choose an action

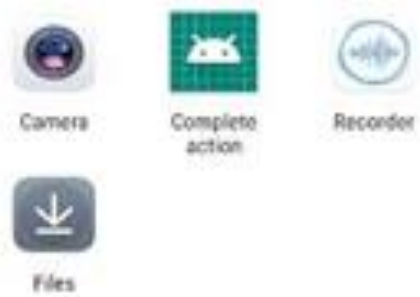

Fig 2:- Accessing phone camera

The live image captured for recognition is shown as in the figure 3 


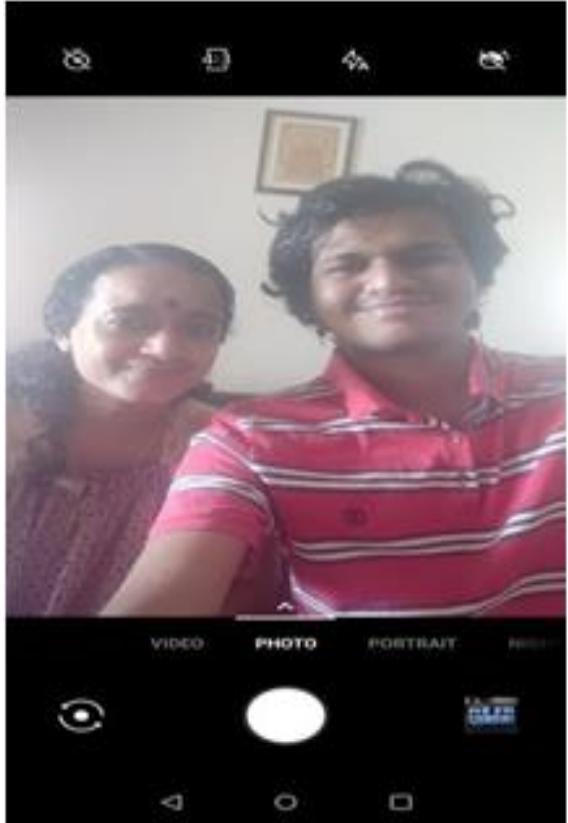

Fig 3:- captured image

\section{Upload new File}

\section{Choose File IMG_202007 123804.jpg Upload}

Fig 4:- uploading the image

\section{Face Detection}

The uploaded image is given to the pretrained classifier, that is the haarcascade frontal face detector for the face detection process. It is a Haar feature based cascade classifier proposed by Paul Viola and Michael Jones [5]. This classifier is trained from a lot of positive(images of faces) and negative images(images without faces) and then used to detect objects in other images. They uses some windows as shown in figure $\mathbf{5}$ to identify the facial features.

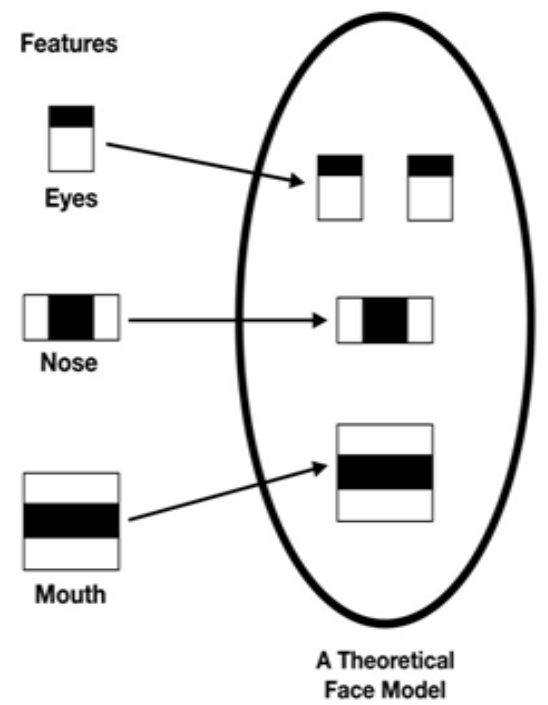

Fig 5:- Haar Cascade Frontal Face Detector
In this cascade of classifier instead of applying all features on a single window, it groups the features into different stages of classifiers and then applied one by one. The first few stages of the classifier contain very less features and if a window in this stage fails, then remaining features are not considered. If it passes, the second stage of features are applied and this process is continued as shown in figure 6. For the detected frontal faces their face embeddings are created using facenet which is then used for face recognition[6][9].

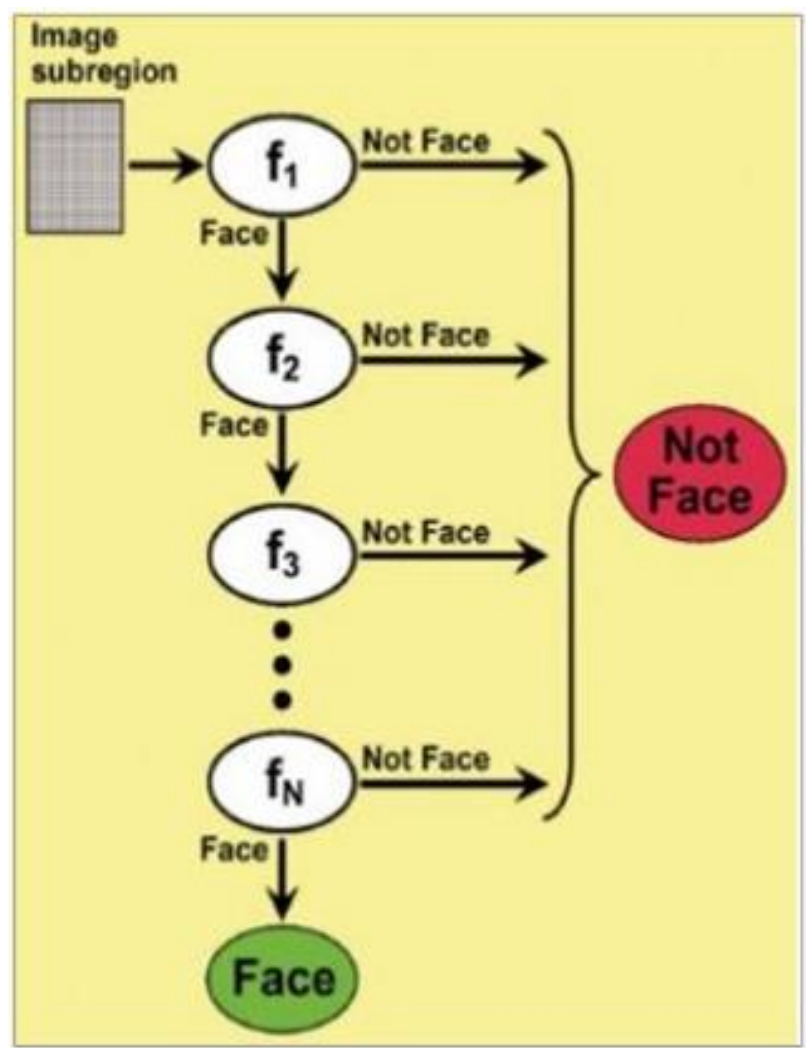

Fig 6:- Face Detection Process

\section{Face recognition}

For the training process, 40 to 50 frontal face images of each person are used. Here we used images of 7 persons for training purpose as shown in figure 7 .

These images are converted into face embedding using facenet[6][9] and trained using neural network.

This trained model weights is then used for face recognition. Here $67 \%$ of total image is used for training and rest is used for testing. Detected face embeddings of the input image is then predicted from trained model for recognition .Faces with similar values are identified as same persons. 


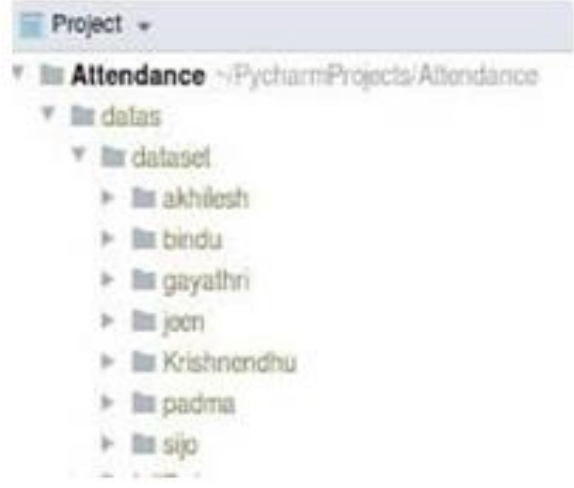

Fig 7:- Folder with trained mages

\section{$>$ Marking attendance onto the excel}

After the process of face detection,the embedding values are then used to predict whether the person on the input image and the trained dataset are similar. In the face recognition process the known and unknown persons are marked as present or absent in the excel sheet.In the Figure $\mathbf{8}$ we can see that the faces are recognized and marked properly.

\begin{tabular}{|c|c|c|}
\hline & Name & Attendance \\
\hline 0 & Krishnendhu & absent \\
\hline 1 & gayathri & absent \\
\hline 2 & bindu & absent \\
\hline 3 & padma & present \\
\hline 4 & sijo & absent \\
\hline 5 & jeen & absent \\
\hline 6 & akhilesh & present \\
\hline
\end{tabular}

Fig 8:- Attendance Marking

\section{SYSTEM SPECIFICATIONS}

This method uses neural network for training .The computer language to be used is python. The platform used is anaconda and flask server. Flask is a web framework that provides with tools ,libraries and technologies that helps to build a web application in python.

\section{RESULT}

Input has been taken from the mobile phone camera which helps in the real time attendance management.Faces from the input image are identified using haar features and it is converted to face embedding. In training process at first the imaes are converted to face embedding from which $67 \%$ of total image are used for training and rest is used for testing purpose.During the recognition process these embeddings are compared and the faces are recognized the attendance is successfully marked in the excel sheet.

\section{CONCLUSION}

This method of Real-time attendance marking system based on deep learning is to overcome the disadvantages of the previous works which include the delay of the processor, accuracy, etc. Deep learning extracts the features of the input dataset and this input dataset is trained using the neural network and this can be done using different deep learning platforms such as Keras, tensor flow and so on. The machine learning program can be done using a platform called Anaconda. Anaconda is free and open source distribution of the python and $\mathrm{R}$ programming languages for machine learning etc. Anaconda provides a flask server where the server program combined with face recognition model is deployed.Using the server IP address, the browser connects mobile phone to the web server. In this attendance marking system, the live images are taken using mobile phone camera via server and then uploaded for face detection and recognition. In this method images are converted into face embedding and prediction is done. Known and unknown faces are then marked as present and absent onto an excel sheet.

\section{REFERENCES}

[1]. M. Yadav, A. Aggarwal and N. Rakesh, "Motion Based Attendence System in Real- Time Environment for Multimedia Application," 2018 8th International Conference on Cloud Computing, Data Science \& Engineering (Confluence), Noida, 2018, pp. 332-336.

[2]. X. Qu, T. Wei, C. Peng and P. Du, "A Fast Face Recognition System Based on Deep Learning," 2018 11th International Symposium on Computational Intelligence and Design (ISCID), Hangzhou, China, 2018, pp. 289-292

[3]. T. Mantoro, M. A. Ayu and Suhendi, "Multi-Faces Recognition Process Using Haar Cascades and Eigenface Methods," 2018 6th International Conference on Multimedia Computing and Systems (ICMCS), Rabat, 2018, pp. 1-5.

[4]. M. Arsenovic, S. Sladojevic, A. Anderla, and D. Stefanovic, "FaceTime — Deep learning-base face recognition attendance system," 2017 IEEE 15th International Symposium on Intelligent Systems and Informatics (SISY), Subotica, 2017, pp. 000053000058.

[5]. P. Viola and M. Jones, "Rapid object detection using a boosted cascade of simple features," Proceedings of the 2001 IEEE Computer Society Conference on Computer Vision and Pattern Recognition. CVPR 2001, Kauai, HI, USA, 2001, pp. I-I, doi: 10.1109/CVPR.2001.990517.

[6]. F. Schroff, D. Kalenichenko and J. Philbin, "FaceNet: A unified embedding for face recognition and clustering," 2015 IEEE Conference on Computer Vision and Pattern Recognition (CVPR), Boston, MA, 2015, pp. 815-823, doi: 10.1109/CVPR.2015.7298682. 
[7]. G. Lou and H. Shi, "Face image recognition based on convolutional neural network," in China Communications, vol. 17, no. 2, pp. 117-124, Feb. 2020, doi: 10.23919/JCC.2020.02.010.

[8]. Z. Yu, F. Liu, R. Liao, Y. Wang, H. Feng and X. Zhu, "Improvement of Face Recognition Algorithm Based on Neural Network," 2018 10th International Conference on Measuring Technology and Mechatronics Automation (ICMTMA), Changsha, 2018, pp. 229-234, doi: 10.1109/ICMTMA.2018.00062.

[9]. William, D. R. Ignatius Moses Setiadi, E. H. Rachmawanto, H. A. Santoso and C. A. Sari, "Face Recognition using FaceNet (Survey, Performance Test, and Comparison)," 2019 Fourth International Conference on Informatics and Computing (ICIC), Semarang, Indonesia, 2019, pp. 1-6, doi: 10.1109/ICIC47613.2019.8985786.

[10]. F. Schroff, D. Kalenichenko and J. Philbin, "FaceNet: A unified embedding for face recognition and clustering," 2015 IEEE Conference on Computer Vision and Pattern Recognition (CVPR), Boston, MA, 2015, pp. 815-823, doi: 10.1109/CVPR.2015.7298682.

[11]. W. Min, M. Fan, J. Li and Q. Han, "Real-time face recognition based on pre-identification and multi-scale classification," in IET Computer Vision, vol. 13, no. 2, pp. 165-171, 32019. 